\title{
Frying Stability of Moringa stenopetala Seed Oil
}

\author{
STAVROS LALAS,,${ }^{1, *}$ OLGA GORTZI $^{1} \&$ JOHN TSAKNIS $^{2}$ \\ ${ }^{1}$ Department of Food Technology, Technological Educational Institution (T.E.I.) of Larissa (Karditsa Branch), Terma N. Temponera str., Karditsa, \\ GR-43100, Greece; ${ }^{2}$ School of Food Technology and Nutrition, Technological Educational Institution (T.E.I.) of Athens, Ag. Spiridonos str., Athens, \\ Greece (*authorfor correspondence; e-mail: slalas@teilar.gr)
}

Published online: 28 June 2006

Abstract. The frying performance of Moringa stenopetala seed oil (extracted with cold press or $n$-hexane) was studied especially as regards repeated frying operations. The oils were used for intermittent frying of potato slices and cod filets at a temperature of $175^{\circ} \mathrm{C}$ for 5 consecutive days (5 fryings per day). The chemical changes occurring in oils were evaluated. Free fatty acid content, polar compounds, colour and viscosity of the oils all increased, whereas the iodine value, smoke point, polyunsaturated fatty acid content, induction period and tocopherol content decreased. The effect of the oil on the organoleptic quality of these fried foods and the theoretical number of frying operations possible before having to discard the oil was also determined. The analytical and sensory data showed that the lowest deterioration occurred in cold press produced oil.

Key words: Cod, Frying stability, Moringa stenopetala, Potatoes, Seed oil

\section{Introduction}

Deep-fried food and especially fried potatoes and cod (the most common foods fried in home and restaurants) are becoming more and more popular among the inhabitants of the European Union. Deep-frying is also a very important method of cooking in the food services industry as it enhances the sensory properties of foods [1].

Repeated use of frying oils produces undesirable constituents that may pose health hazards. In view of this, the use of oil that can resist as much as possible the oxidation is very important. Virgin olive oil has proven its oxidative stability during frying [2] which is related to some extent to the presence of $\alpha$-tocopherol [3] and phenolic compounds [4]. However, its applications in food industry are limited due to its high cost. The use of an oil with similar characteristics to olive oil but equal or increased resistance to oxidation during frying would be most appreciated.

In previous work [5], we reported that the oil produced from the seeds of Moringa stenopetala had similar level of $\mathrm{C}_{18: 1}$ to olive oil. The same oil showed an increased, up to 6 times, induction period (as determined by the Rancimat method) in comparison to virgin olive oil.

This work tries to investigate the possible use of the oil produced (with cold press or solvent extraction) from the seeds of Moringa stenopetala as frying oil. The physical and chemical changes that occur during frying potatoes and cod where determined. The possible relevance of minor compounds in the frying life of the oil is being discussed.
Finally, the theoretical number of frying operations possible before having to discard the oil was calculated.

\section{Materials and Methods}

\section{Materials}

Moringa stenopetala (variety Marigat from the island Kokwa) seeds were assayed from Kenya Forestry Research Institute (KE.F.R.I., Nairobi, Kenya). Thirty kilograms of seeds were harvested, air-dried for one week, mixed well and divided in three individual portions of $10 \mathrm{~kg}$ each. Potatoes (variety Lady Rosetta) and cod fillets (Gadus morhua) were purchased from the local market.

\section{Reagents}

All solvents (analytical and HPLC grade) were obtained from Sigma Chemicals Company Co. (St. Louis, MO 63178, USA) and the tocopherol standards from Merck Ltd (Darmstand, D-64271, Germany) ( $\alpha$-tocopherol) or Sigma ( $\delta$-tocopherol). Fatty acid methylester standards were purchased from British Greyhound Chromatography and Allied Chemicals (Birkenhead, Merseyside, L434X, UK).

\section{Oil Extraction and Degumming}

Oil extraction was carried out using the method described by Lalas et al. [5]. The seeds were divided onto two portions for cold press and solvent extraction (using $n$-hexane). Degumming was carried out using the method also described by Lalas et al. [5].

\section{Determination of the Physical and Chemical Characteristics}

The method used for the determination of density and refractive index (at $40{ }^{\circ} \mathrm{C}$ ) was adapted from AOAC (method number 969.18) [6]. Colour was measured with a Lovibond tintometer (The Tintometer Ltd., Salsbury, England). Acidity was measured according to IUPAC (method number 2.201) [7], saponification value according to Sonntag [8], 
and iodine value according to the Wijs method as described by Pearsons [9].

\section{Determination of the Fatty Acid Composition}

Fatty acid composition was determined by gas-liquid chromatography according to the method of Lalas et al. [5]. The analysis was performed on a Varian 3600 Gas chromatograph (Varian, Palo Alto, California, USA) equipped with a Supelcowax 10 (Supelco, INC., Supelco Park, Bellefonte, PA 16823-0048) fused silica capillary column $30 \mathrm{~m}$ $\times 0.32 \mathrm{~mm}$ ID, $0.25 \mu \mathrm{m}$ film thickness.

\section{Determination of the Tocopherol Composition}

The method used for the determination of tocopherols was adapted from Tsaknis et al. [10]. A Waters $\mu$-Porasil (125 ^, $10 \mu \mathrm{m}, 3.9 \mathrm{~mm} \times 300 \mathrm{~mm}$ ) column fitted to a Waters $600 \mathrm{E}$ HPLC pump (Millipore Corporation, Waters Chromatography Division, Massachusetts, MA 01757, USA), equipped with a Waters 486 Tunable Absorbance Detector, was used.

\section{Determination of the Susceptibility}

to Oxidation (Rancimat Method)

The determination of the susceptibility to oxidation (Rancimat method) was carried out using the method described by Lalas et al. [5].

\section{Polar Compounds}

They were determined using the IUPAC method [7].

\section{Frying}

The frying method used was adapted from Tsaknis and Lalas [11]. Potatoes and skinless cod fillets were deep-fried independently, each in 21 of oil.

The potatoes were peeled and washed about $1 \mathrm{~h}$ before use, and sliced into discs $0.5 \mathrm{~cm}$ thick and $2.5 \mathrm{~cm}$ in diameter, using a mechanical slicer. The skinless cod fillets were cut into square pieces $(3 \mathrm{~cm} \times 3 \mathrm{~cm} \times 1.5 \mathrm{~cm})$ and coated with wheat flour. When the temperature of the oil reached $175^{\circ} \mathrm{C}$ a batch of $100 \mathrm{~g}$ of each product (potatoes or cod) were fried in separate oil samples. The temperature of the oil during frying was $175 \pm 5^{\circ} \mathrm{C}$.

Repeated fryings were carried out at half-hour intervals for $2 \mathrm{~h}$. The frying time was $8 \mathrm{~min}$ for cod fillets and $6 \mathrm{~min}$ for potatoes. A total of five fryings were done per day for five consecutive days (25 fryings in total).

At the end of each day, $50 \mathrm{~g}$ sample of oil was removed from each fryer and stored at $0{ }^{\circ} \mathrm{C}$. The lid of the fryer was replaced and the fryings were continued the following day. Fresh oil was not added to the frying pans.

\section{Sensory Evaluation}

The method used was adapted from Tsaknis and Lalas [11]. 8 panellists, members of the sensory panel, were used. Fish and potatoes were kept for $10 \mathrm{~min}$ at room temperature before testing. In the beginning, the panellists were trained to use the triangular test in order to distinguish the difference in the organoleptic properties (colour, off-flavour) of the fried products. The samples used were fried either with fresh oil or with oil previously used for 10 separate fryings beforehand. Assessment was conducted in natural white light in a specially designed taste panel room. A taste panel score sheet with a numerical scale of rating was developed, using descriptive terms against each quality parameters as follows: colour (only for fried potatoes because the panellists were not able to detect any difference in colour of cod samples during training sessions) scale of 1 to 4 , an increasing score corresponding to decreasing quality, and a score of 4 represented extremely undesirable colour. Offflavour, scale of 1 to 4 , a score of 1 represented "no rancid off-flavour" and a score of 4 represented "strong rancid off-flavour." In the overall acceptance scale of 1 to 9, the two extreme ends represent conditions of quality, either extremely good (1) or totally undesirable (9). The panellists were provided with the score sheets and were asked to rate samples according to the descriptive scale. One sample from each frying pan (potatoes and cod) was represented at one session to each panellist. The hot samples were served in shallow disposable polystyrene food dishes with a code symbol for identification inscribed on the dish against each sample. In order to avoid transfer (carry over) of flavour components from one sample to the next, the panellists rinsed their mouths with water between tastings. Each day the panellists tasted 20 products $(2$ oils $\times 2$ products $\times 5$ fryings).

\section{Statistical Analysis}

Results are representing the average and the standard deviation (SD) (in parenthesis) of three simultaneous assays carried out in all methods. Changes in polar compound content were adjusted to linear $(Y=a+b X)$ adjustment equation by analysis of variance (ANOVA) $(P<0.05)$, where $Y=$ polar compounds and $X=$ hours of frying. Using the linear equation and having as limit the $25 \%(\mathrm{w} / \mathrm{w})$ of polar compounds, the theoretical number of frying hours possible before having to discard the oil was calculated.

\section{Results and Discussion}

Fish and potatoes are the most commonly fried foods in homes and restaurants. In view of this, the use of an oil with improved resistance to oxidation and level of 
unsaturation, as higher as possible, would be the most desirable. An investigation was carried out to study the physical and chemical changes which occur in oil produced (with cold press or solvent extraction) from the seeds of Moringa stenopetala used for frying.

\section{Free Fatty Acid (FFA) Content}

No significant increases were observed after 25 fryings in all the oils at the $95 \%$ level of significance (student's $t$ test) (Tables 1-4). The cold press oil showed the lowest increase. The steady rise in the formation of FFA can be attributed partly to the hydrolysis and partly to the component carboxylic groups present in polymeric products of frying $[2,12]$. The acidity was mainly formed by hydrolysis of triglycerides, which was promoted by the presence of food moisture, and by oxidation or by the reaction of oil with moisture formed during other deterioration reactions $[12,13]$. The oils used for frying cod seemed to follow the same trend. Although, cod was found to cause a more rapid increase in the free fatty acid content.

\section{Iodine Value}

There were no significant changes $(P<0.05)$ between the fresh and used oils after 25 fryings (Tables 1-4). The analysis confirmed that less oxidation of unsaturated fatty acids has taken place in cold press oil. The oils used for frying cod seemed to follow the same trend but with a more rapid decrease in the iodine value.

\section{Viscosity}

As the oxidation is accelerated by heat proceeded, the values of viscosity progressively increased [12]. Cold press oil showed the lowest change in viscosity after 25 fryings, while $n$-hexane oil showed much higher changes (significant at $P<0.05$ ) (Tables $1-4$ ). These results clearly indicated the higher deteriorative effect of oxidation and polymerisation of $n$-hexane oil compared to cold press oil. The same results were observed by Tsaknis and Lalas [11] during frying in $n$-hexane extracted Moringa oleifera seed oil. The increase in viscosity of frying oils was due to polymerisation that resulted in formation of higher molecular weight compounds (carbon to carbon and/or carbon to oxygen-to carbon bridges) between fatty acids $[11,13]$. The oils used for frying cod seemed to follow the same trend but again with a more rapid increase in viscosity.

\section{Polar Compounds}

The results demonstrated (Tables 1-4) that cold press oil exhibited the lowest increase (significant at $P<0.05$ ) in polar compounds. The analysis of percentage polar compounds is considered to be one of the more reliable indicators of the state of the oil deterioration [11].

As indicated by Romero et al. [14] and Tsaknis and Lalas [11] a number of European countries have passed specific laws and regulations concerning culinary oil use in frying. Many countries have set polar compounds maximum level at $25 \%(\mathrm{w} / \mathrm{w})$ while others have established a polar compound cut point between 20 and 27\%. Romero et al. [14], in their work, used three different equations, namely linear, logarithmic and power, to estimate the number of frying operations before the critical quantity of polar compounds was reached during frying (frying oil was frequently replenished with fresh at a quantity lost during operations). Logarithmic and power equations appeared to define the changes more accurately because polar compounds tended to stabilise after certain number of fryings. Tsaknis and Lalas [11] used only the linear equation as the more adequate because no replenishment with fresh oil during fryings took place and the polar compounds kept increasing until the end of fryings. This also applies to our situation.

The theoretical frying hours until the polar compounds reach the critical level of $25 \%(\mathrm{w} / \mathrm{w})$, using the linear equation $Y=a+b X$ were: $Y=3.4893+0.4783 X$ (potatoes and cold press oil), $Y=2.3033+0.9357 X$ (potatoes and $n$-hexane oil), $Y=3.3507+0.5931 X(\mathrm{cod}$ and cold press oil) and $Y=2.1887+1.0094 X$ (cod and $n$-hexane oil). According to the above equations, the hours needs to reach the critical level before having to discard the oil were calculated as 45 (potatoes) or 37 (cod) hours of frying for cold press oil and 24 (potatoes) or 23 (cod) hours of frying for $n$-hexane oil.

\section{FAME Analysis}

There was a decrease in polyunsaturated fatty acids and a resulting increase in the saturated acid content (Table 5. Results are displayed only for cold press oil during frying potatoes). However, the changes in polyunsaturated fatty acids were not statistically significant $(P<0.05)$ apart from the case of $\mathrm{C}_{18: 3}$. The same results were observed by Tsaknis and Lalas [11] during frying of Moringa oleifera seed oil. Changes in fatty acid profile of all oils during frying are basically among the unsaturated fatty acids, whereas the saturated fatty acids (myristic, palmitic and stearic) were slightly increased [12].

\section{Colour}

Darkening is attributed to the presence of unsaturated carbonyl compounds or to non-polar compounds of foodstuff solubilised in the oil [15]. The results (Tables 1-4) indicated that there was an increase in red units of the colour measurement of frying oils, while yellow units showed only minor changes. The colour change was a result of the diffusion of 


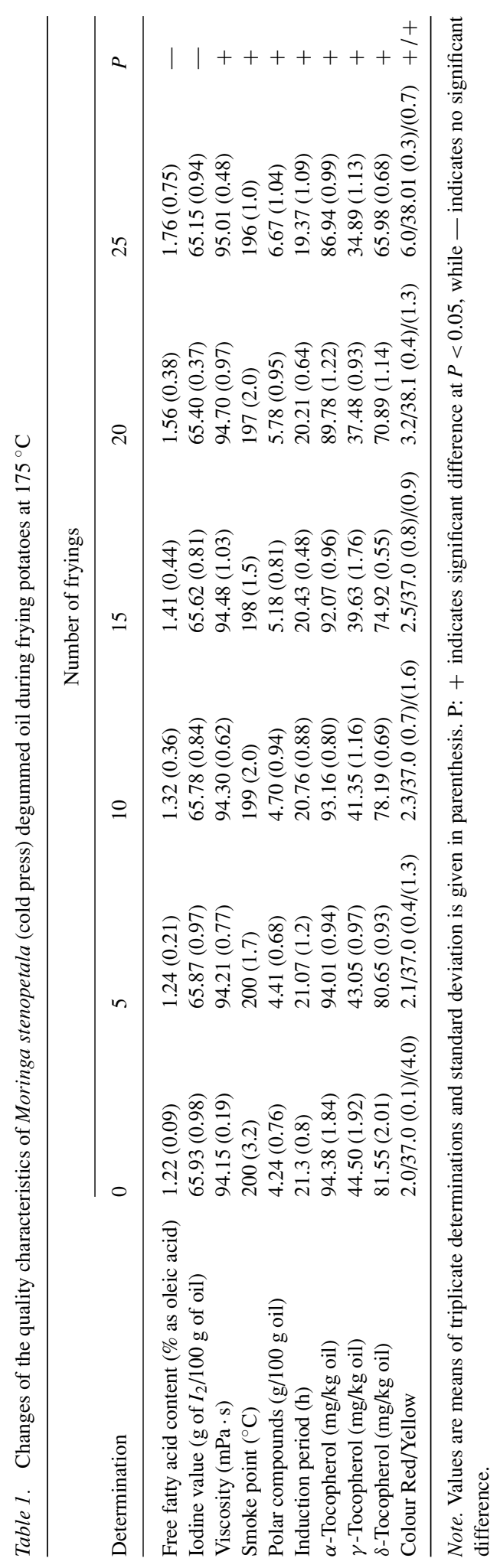




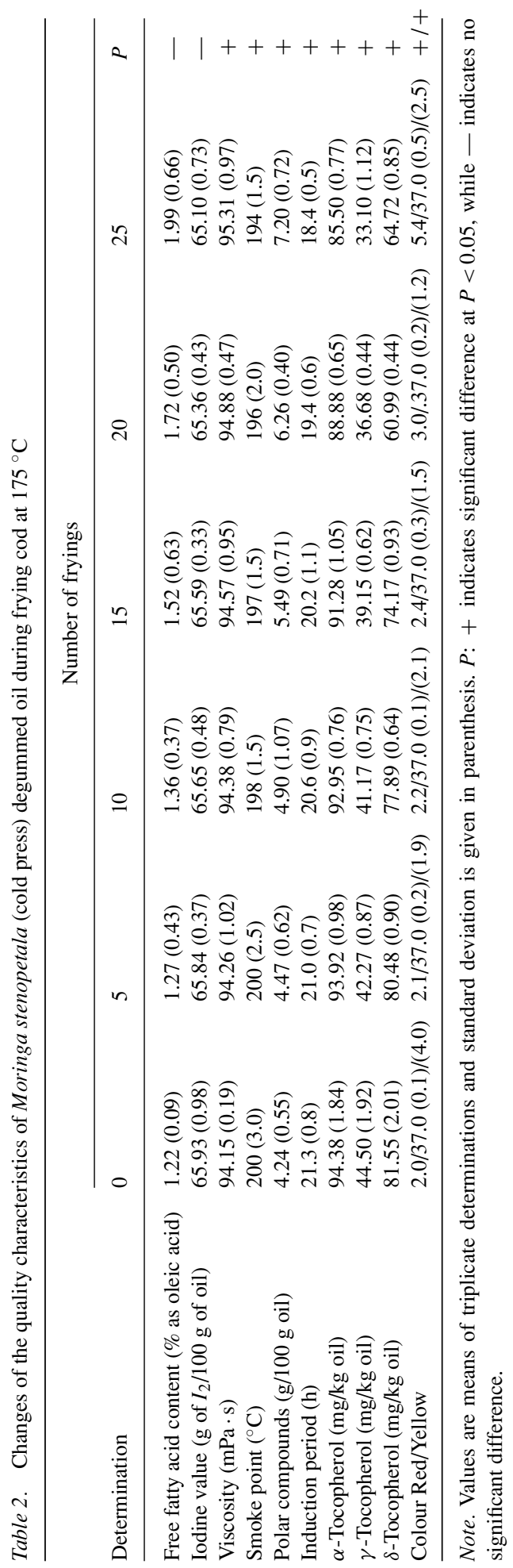




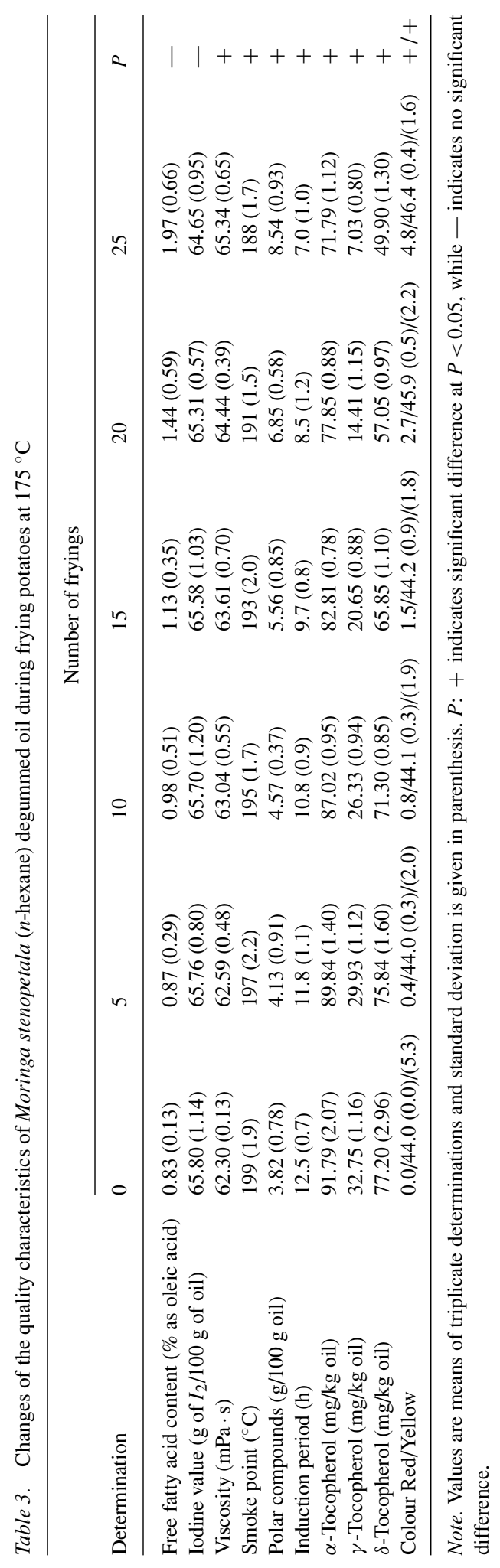




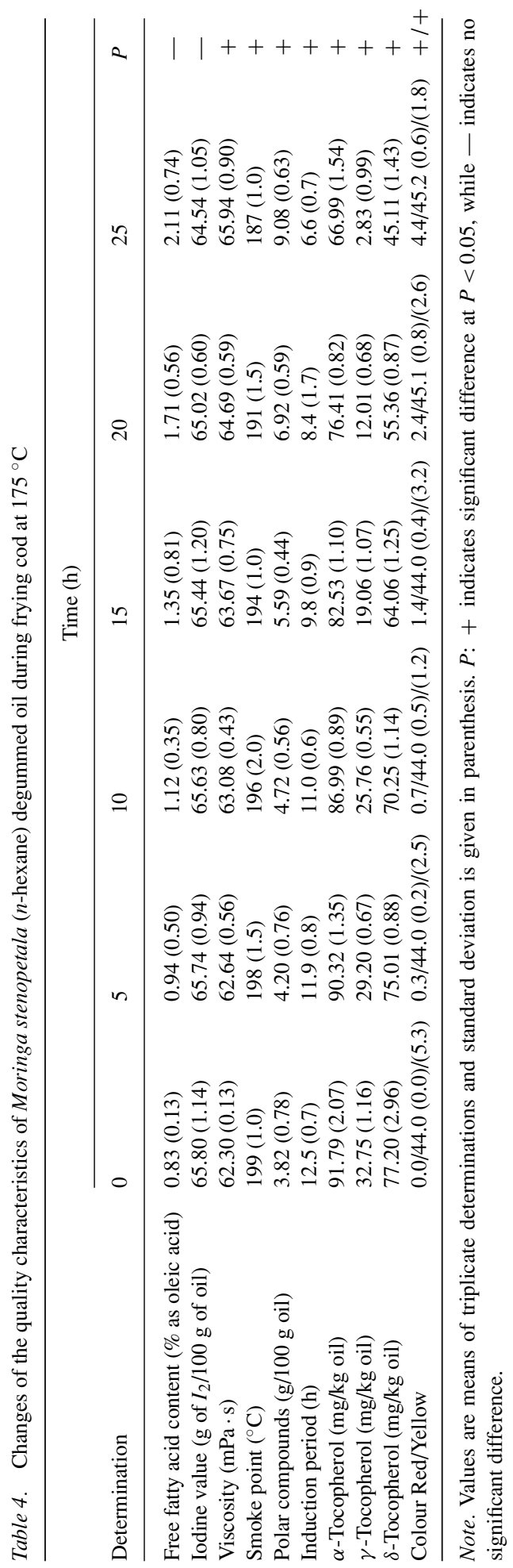




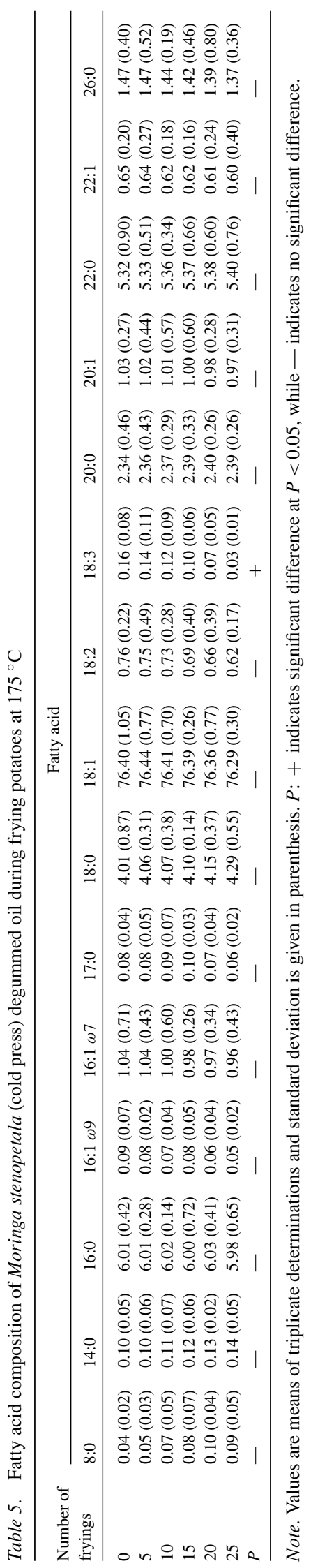


pigments into the oil during frying. $n$-Hexane oil showed the highest increase in colour while cold press oil showed the lowest. Although, cod was found to cause less increase in red units of colour. The higher increase of oil colour during frying of potatoes is due to the fact that reactions between the aldehyde group of sugar and amino acids give brown products [11]. Burton [16] reported that the alpha, beta unsaturated carbonyl compounds, derived from the sugars are the first formed intermediates that react with substances containing alpha-amino groups to give carbonyl-nitrogen compounds which conjugate to form brown products.

\section{Tocopherols}

The relative decomposition rates after 25 fryings were $\delta>\gamma>\alpha$ (Tables 1-4). The same results were observed by Tsaknis and Lalas [11] during frying of Moringa oleifera seed oil. The results are also in agreement with those of Sonntag [17], who reported that the decomposition rates of tocopherols, after $10 \mathrm{~h}$ frying, were $\gamma>\alpha$.

\section{Induction Period}

All oils showed a significant $(P<0.05)$ decrease in induction period after 25 fryings (Tables $1-4)$. Induction period measurements were carried out on frying oils in order to provide a quick indication of the trends in resistance to oxidative rancidity of the heated oils. The induction period determined via accelerated oxidation methods on the original oil cannot guarantee or predict the actual flying performance of the oil as other factors will be introduced once frying commences (e.g. badly operated fryer or heat exchanger will ruin even the best quality oil) [11]. Nevertheless, it is considered that the "Rancimat" induction period can be useful to act as a "screening" test and eliminate the possibility of introducing lower stability oils into the production area with all the attendant consequences [18]. The oils used for frying cod seemed to follow the same trend. Although, again cod was found to cause a higher decrease in induction period.

The oils used for frying cod, showed higher deterioration in all the quality parameters, with the exception of colour, compared with the same oils used for frying potatoes. This greater deterioration could be due to the leaching of fish oil from the cod, which is rich in polyunsaturated fatty acids, which are rapidly oxidised [11].

\section{Sensory Evaluation}

A number of panellists were chosen to taste fried products (potatoes and cod) for the overall characterisation of the organoleptic properties after each batch had been fried. A taste panel score sheet with a numerical scale of rating was developed, using descriptive terms against each nu- merical score for each quality parameter. Throughout the frying time the overall acceptance scores showed a significant difference in overall acceptance of fried potatoes with cold press oil after 25 fryings, while this difference was significant after 20 fryings with $n$-hexane oil (results not displayed). The overall acceptance scores showed that fried cod was unacceptable after having been fried in oil for 5 days in cold press oil and after 3 days in $n$-hexane oil. The panel scores confirmed that cold press oil was the most suitable oil for repeated frying. Tsaknis and Lalas [11] reached to the same result during frying of Moringa oleifera seed oil. So, it appears that the oils extracted from the seeds of the two kinds of Moringa (stenopetala and oleifera) present the same behaviour during frying.

In conclusion, the changes in characteristics and composition of used oils showed that cold press oil was more stable during frying and can be used as frying oil in food applications. Additionally, since its level of unsaturation resembles to olive oil, it can became a possible substitute.

\section{Acknowledgments}

This research was funded by European Union (CEC contract No: TS3 ${ }^{*}$ CT-94-0399 for Developing Countries).

\section{References}

1. Lalas S, Dourtoglou V (2003) Use of rosemary extract in preventing oxidation during deep fat frying of potato chips. J Am Oil Chem Soc 80: 579-583.

2. Chatzilazarou A, Gortzi O, Lalas S, Zoidis E, Tsaknis J (2006) Physicochemical changes of olive oil and selected vegetable oils during frying. J Food Lipids 13: 27-35.

3. Kiritsakis A (1989) Tocopherols. In: Kafatos A (ed), The Olive Oil. Thessaloniki, Greece: Agricultural Co-operative Editions, pp 61-64 and 234.

4. Kiritsakis A, Min DB (1989) Flavour chemistry of olive oil. In: Min DB (ed) Flavour Chemistry of Lipid Foods. Illinois, USA: The American Oil Chemists' Society, pp 196-221.

5. Lalas S, Tsaknis J, Sflomos K (2003) Characterisation of Moringa stenopetala seed oil variety "Marigat" from island Kokwa. Eur J Lipid Sci Tech 105: 23-31.

6. AOAC (1990) Official Methods of Analysis, 15th edn. Arlington, Virginia, USA: Association of Official Analytical Chemists.

7. Standard methods for the analysis of oils, fats and derivatives (1987) In: Paquot C, Hautfenne A (eds), International Union of Pure and Applied Chemistry (IUPAC), 7th edn. London, UK: Blackwell Scientific Publications, pp 212-213.

8. Sonntag NOV (1982) Analytical methods. In: Swern DE (ed) Bailey's Industrial Oil and Fat Products 2, 4th edn, New York, USA Wiley, pp 407-527.

9. Pearsons D (1981) Chemical Analysis of Foods, 8th edn. Edinburgh, UK: Churchill Livingstone.

10. Tsaknis J, Spiliotis V, Lalas S, Gergis V, Dourtoglou V (1999) Quality changes of Moringa oleifera, variety Mbololo of Kenya seed oil during frying. Grasas Aceites 50: 37-48. 
11. Tsaknis J, Lalas S (2002) Stability during frying of Moringa oleifera seed oil variety "Periyakulam 1." J Food Compos Anal 15: 79-101.

12. Tyagi VK, Vasishtha AK (1996) Changes in characteristics and composition of oils during deep fat frying. J Am Oil Chem Soc 73: 499-506.

13. Al-Harbi MM, Al-Kabtani HA (1993) Chemical and biological evaluation of discarded frying palm oil commercial restaurants. Food Chem 48: 395-401.

14. Romero A, Cuesta C, Sanchez-Muniz FJ (1999) Does frequent replenishment with fresh monoenoic oil permit the frying of potatoes indefinitely? J Agr Food Chem 47: 1168-1173.
15. Gutierrez R, Gonzalez O, Dobarganes MC (1988) Analytical procedures for the evaluation of used frying fats. In: Varela G, Bender AE, Morton ID (eds) Frying Food: Principles, Changes, New Approaches, London, UK: VCH Publishers Ltd, pp 141-154.

16. Burton WG (1989) The Potato, 3rd edn. New York: Wiley.

17. Sonntag NOV (1991) Erucic, behenic: Feedstocks of the 21st century. Inform 2: 449-463.

18. Morton ID, Chidley JE (1988) Methods and equipment in frying. In: Varela G, Bender AE, Morton ID (eds) Frying Food: Principles, Changes, New Approaches, London, UK: VCH Publishers Ltd, pp $37-51$. 